\title{
AUTHOR INDEX Volume 18
}

Abbaspur, R., Generalized noncommutative superalgebras Abdalla, E., see Wang

Abdalla, E., Castello-Branco, K.H.C. \& Lima-Santos, A., Area quantization in quasiextreme black holes

Abdelrahman, Y.S., see Rabei

Abou-Shady, H., see Ismail

Abramo, L.R., Brenig, L., Gunzig, E. \& Saa, A., A note on dualities in Einstein's gravity in the presence of a non-minimally coupled scalar field

Abrikosov, Jr., A.A., Gozzi, E. \& Mauro, D., Time and geometric quantization

Accioly, A. \& Blas, H., Conformal coupling and FoldyWouthuysen transformation

Adam, J., Jr., see Yuan

Agarwal, A. \& Akant, L., Gauge theories on open Lie algebra noncommutative spaces

Aguado, M., Asorey, M. \& García-Alvarez, D., $\theta$-physics: 0 -vacuum versus $\pi$-vacuum

Ahmed, M.W., see Weller

Aizawa, I., see Hayashi

Aizawa, N., Chakrabarti, R. \& Segar, J., Jordanian quantum superalgebra $U_{h}(\operatorname{osp}(2 / 1))$

Ajimura, S., see Tamura

Akaishi, Y., see Doté

Akaishi, Y., see Shinmura

Akaishi, Y., see Nemura

Akant, L., see Agarwal

Aketo, I., see Hayashi

Akikawa, H., see Tamura

Akiyoshi, H., see Yagita

Alburger, D.E., see Tamura

Amézaga, A. \& Leiva, C., Algebra of chiral currents on the physical surface

Andrianopoli, L., D’Auria, R., Ferrara, S. \& Lledó, M.A., Gauged extended supergravity without cosmological constant: No-scale structure and supersymmetry breaking
A18(2003) 587
A18(2003) 31

A18(2003)1435

A18(2003) 1591

A18(2003) 57

A18(2003)1043

A18(2003)2347

A18(2003) 867

A18(2003)244

A18(2003)491

A18(2003)2303

A18(2003) 1569

A18(2003)2785

A18(2003) 885

A18(2003) 85

A18(2003) 120

A18(2003) 128

A18(2003) 139

A18(2003)491

A18(2003) 2785

A18(2003) 85

A18(2003) 322

A18(2003) 85

A18(2003)609

A18(2003)1001
Anselmo, F., Ellis, J., Volkov, G. \& Nanopoulos, D.V., Universal Calabi-Yau algebra: Classification and enumeration of fibrations

A18(2003)699

Aoyama, S., see Myo

A18(2003) 166

Aoyama, S., Analyses of threebody $S$-wave resonances with analytic continuation in the coupling constant

Aoyama, S. \& Masuda, T., Fuzzy algebras of the general Kähler coset space $G / S \otimes \mathrm{U}(1)^{k}$

Arenhövel, H., Darwish, E.M., Fix, A. \& Schwamb, M., Present status of electromagnetic reactions on the deuteron above pion threshold

Asorey, M., see Aguado

Aydemir, A., see Sever

Azam, M., An exact equation for Wilson loops in two-dimensional Euclidean space

Bai, J., see Zhang

Baldo, M., see Song

Banu, A., see Tamura

Bao, C.G., see Liu

Barbieri, J., Chapline, G. \& Santiago, D.I., Quantum criticality, event horizons and cosmic gamma ray bursts

Barros, A. \& Romero, C., Gravitomagnetic time delay and the Lense-Thirring effect in Brans-Dicke theory of gravity

Barros, A., Bezerra, V.B. \& Romero, C., Global aspects of gravitomagnetism

Barshay, S. \& Kreyerhoff, G., Very high-energy neutrinos from slowly decaying, massive dark matter, as a source of explosive energy for gamma-ray bursts

Barshay, S. \& Kreyerhoff, G., Final-state interactions in coupled channels of physical hadrons resulting in a sizable, direct CP-violation in $B^{0}\left(\bar{B}^{0}\right) \rightarrow \pi^{+} \pi^{-}$
A18(2003) 2117

A18(2003)422

A18(2003)553

A18(2003) 190

A18(2003)2303

A18(2003)545

A18(2003)1925

A18(2003)151

A18(2003)317

A18(2003) 85

A18(2003)414

A18(2003)2767

A18(2003)2673

A18(2003)477, 875(E)

A18(2003)2887 
Battistel, O.A. \& Krein, G., Quark clustering and chiral symmetry breaking in nuclear matter

Bennhold, C., see Miyagawa

Bentz, W., see Mineo

Bergman, D.R., Chasing the GZK with HiRes

Berman, B.L. (for the CLAS Collab.), Photoreactions on ${ }^{3} \mathrm{He}$ and ${ }^{4} \mathrm{He}$ up to $1.6 \mathrm{GeV}$ at Jefferson Lab

Besprosvany, J., Standard-model coupling constants from compositeness

Bezerra, V.B., see Barros

Bhattacharyya, R. \& Gangopadhyay, D., Vacuum expectation value of the Higgs field and dyon charge quantisation from spacetime dependent Lagrangians

Bhattacharyya, S., see De

Bhattacharyya, S., see Ghosh

Bhowmick, S., see Sinha

Bimonte, G., Esposito, G., Marmo, G. \& Stornaiolo, C., Classical brackets for dissipative systems

Blas, H., see Accioly

Błaut, A., Daszkiewicz, M. \& Kowalski-Glikman, J., Doubly special relativity with lightcone deformation

Bobkov, K., Graviton-scalar interaction in the $p p$-wave background

Borowiec, A., Lukierski, J. \& Tolstoy, V.N., Basic twist quantization of $\operatorname{osp}(1 \mid 2)$ and к-deformation of $D=1$ superconformal mechanics

Bose, S.K. \& Schulze-Halberg, A., Improved SUSY inspired variational method to obtain bound state energies of the screened Coulomb potential

Bourrely, C., Buccella, F. \& Soffer, J., Recent tests for the statistical parton distributions

Boz, M. \& Karagöz, N., ${ }^{205}$ Tl electric dipole moment: Strong versus supersymmetric $\mathrm{CP}$ problems
18(2003)2255

A18(2003)290

A18(2003)384

A18(2003)1235

A18(2003)225

A18(2003)1877

A18(2003)2673

A18(2003)2207

A18(2003)1383

A18(2003)2281

A18(2003)661

A18(2003)2311

A18(2003)867

A18(2003)1711

A18(2003)1441

A18(2003)1157

A18(2003)853

A18(2003)771

A18(2003)1601
Brenig, L., see Abramo

Buccella, F., see Bourrely

Buccella, F. \& Falcone, D., Bounds for the mass of the heaviest right-handed neutrino in $\mathrm{SO}(10)$ theories

Burkert, V.D., Spin response of the nucleon in the resonance region

Cai, X.Z., see Zhang

Calian, V. \& Stoenescu, G., Nonlocal regularization and renormalization of $\mathrm{Sp}(2)$ symmetric BRST theories

Cao, L.-M., see Zhang

Capozziello, S., Iovane, G. \& Lambiase, G., Neutrino condensates at center of galaxies as background for the MSW mechanism

Cárdenas, V.H., del Campo, S. \& Herrera, R., $R^{2}$-corrections to chaotic inflation

Carrion, H.L., Rojas, M. \& Toppan, F., Residual symmetries in the presence of an em background

Carrion, H.L., Rojas, M. \& Toppan, F., Octonionic realizations of one-dimensional extended supersymmetries. A classification

Casadio, R. \& Mazzacurati, L., Bulk shape of brane-world black holes

Castello-Branco, K.H.C., see Abdalla

Chakrabarti, R., see Aizawa

Chakrabarty, S., see Ghosh

Chakraborty, N.C., see Chakraborty

Chakraborty, S., Asymptotic behavior of homogeneous cosmological models on the brane

Chakraborty, S., see Debnath

Chakraborty, S., Chakraborty, N.C. \& Debnath, U., Quintessence problem and BransDicke theory

Chan, H.-M., Yang-Mills duality as the origin of fermion generations

Chapline, G., see Barbieri
A18(2003) 1043

A18(2003)771

A18(2003)1819

A18(2003)262

A18(2003)151

A18(2003) 2217

A18(2003)2587

A18(2003)905

A18(2003)2039

A18(2003)629

A18(2003)787

A18(2003)651

A18(2003)1435

A18(2003)885

A18(2003)1297

A18(2003)1549

A18(2003)1197

A18(2003)1265

A18(2003)1549

A18(2003) 537

A18(2003)2767 
Chaturvedi, S. \& Gupta, V., Model of the quark mixing matrix involving its eigenvalues

Chaturvedi, S. \& Gupta, V., Predictions for the unitarity triangle angles in a new parametrization

Chen, H., Beijing electronpositron collider: Results and future plan

Chen, J.G., see Zhang

Chen, J.S., see Meng

Chen, L.X., see Zhang

Chen, S.-X. \& Yang, Z.-Y., Noncommutativity as a possible origin of the ultrahigh energy cosmic ray and the $\mathrm{TeV}$-photon paradoxes

Chen, Z.Q., see Zhang

Cheng, Y. \& Liu, F., Anti-proton to proton ratios from $\mathrm{Au}+\mathrm{Au}$ collisions at $\sqrt{s_{N N}}=200 \mathrm{GeV}$ by RQMD

Chin, C., Kerman, A.J., Vuletić, V. \& Chu, S., Controlled atommolecule interactions in ultracold gases

Chmielewski, K., see Yuan

Chmielewski, K., see Deltuva

Choban, E.A., see Goncharov

Chrien, R.E., see Tamura

Chu, S., see Chin

Chua, C.-K., Hou, W.-S. \& Yang, K.-C., Indication for large rescatterings in charmless rare $B$ decays

Ciftci, H. \& Sultansoy, S., New hadrons formed by the fourth $\mathrm{SM}$ family and iso-singlet quarks

Cirigliano, V., see Maltman

Cleaver, G. \& Tanaka, K., Ratio of quark masses in duality theories

Cooperstock, F.I. \& Faraoni, V., Extended Planck scale

Corradini, O., Iglesias, A. \& Kakushadze, Z., Diluting solutions of the cosmological constant problem

Crowe III, B.J., see Tornow

Crowell, A.S., see Tornow

Czakon, N.G., see Tornow
A18(2003)1635

A18(2003)1825

A18(2003)1141

A18(2003) 151

A18(2003)2029

A18(2003) 151

A18(2003)2913

A18(2003)151

A18(2003)1107

A18(2003)398

A18(2003)244

A18(2003)426

A18(2003)1661

A18(2003) 85

A18(2003)398

A18(2003)1763

A18(2003)859

A18(2003)366

A18(2003)1743

A18(2003)1037

A18(2003)1343

A18(2003) 258

A18(2003) 258

A18(2003)282
D'Ambrosio, G., Recent developments in rare kaon decays

A18(2003) 1273

D'Auria, R., see Andrianopoli

Dąbrowski, L., Krajewski, T. \& Landi, G., Non-linear $\sigma$-models in noncommutative geometry: Fields with values in finite spaces

Dadhich, N., see Singh

Dahia, F., Monte, E.M. \& Romero, C., Fifth force from fifth dimension: A comparison between two different approaches

A18(2003)1773

Daoud, M. \& Douari, J., Extended Weyl-Heisenberg algebra and RubakovSpiridonov superalgebra: Anyonic realizations

A18(2003)1001

A18(2003)2371

A18(2003)983

Darwish, E.M., see Arenhövel

Daszkiewicz, M., see Błaut

Davies, I. \& Mansfield, P., A large- $D$ Weyl invariant string in anti-de Sitter space

de Albuquerque, L.C., deLyra, J.L. $\&$ Teotonio-Sobrinho, P., Fluctuating commutative geometry de S. Pires, C.A., see Gusso de S. Pires, C.A., see PérezLorenzana

De, B. \& Bhattacharyya, S., Transverse mass distribution characteristics of $\pi^{0}$ production in ${ }^{208} \mathrm{~Pb}$-induced reactions and the combinational approach

Deb, A., see Ghosh

Debergh, N., Ndimubandi, J. \& van den Bossche, B., Polynomial deformations of $\operatorname{sl}(2, \nabla)$ in a three-dimensional invariant subspace of monomials

Debnath, U. \& Chakraborty, S., The study of gravitational collapse model in higher dimensional spacetime

Debnath, U., see Chakraborty

Dedes, A., The Higgs penguin and its applications: An overview

Deguchi, S. \& Kokubo, Y., Abelian projection of massive SU(2) Yang-Mills theory del Campo, S., see Cárdenas Deltuva, A., see Yuan
A18(2003)1013

A18(2003) 1265

A18(2003)913

A18(2003)1711

A18(2003) 1517

A18(2003)2517

A18(2003)1849

A18(2003)65

A18(2003) 1383

A18(2003)2281

A18(2003)1549

A18(2003)2627

A18(2003)2051

A18(2003)2039

A18(2003)244
A18(2003)190 
Deltuva, A., Chmielewski, K. \& Sauer, P.U., Nucleon-deuteron scattering with $\Delta$-isobar excitation: New technical developments

deLyra, J.L., see de Albuquerque

Desai, B.R., Roy, D.P. \& Vaucher, A.R., Three-neutrino mass matrices with two texture zeros

Detmold, W., Melnitchouk, W. \& Thomas, A.W., Extraction of parton distributions from lattice QCD

Dey, J., see Sinha

Dey, M., see Sinha

Dolan, B.P., Non-commutative complex projective spaces and the standard model

Donoghue, J.F., see Maltman

Doté, A., Akaishi, Y. \& Yamazaki, T., Systematic studies of stange nuclear systems: Unusual structures formed by a $\mathrm{K}^{-}$meson

Douari, J., see Daoud

Duan, Y.-S., see Zhang

Dulat, S., The $N=2$ superconformal $Z_{3}$ orbifold-prime model with $c=3$

Dzhunushaliev, V. \& Singleton, D., Ginzburg-Landau equation from $S U(2)$ gauge field theory

Dzhunushaliev, V. \& Singleton, D., Effective 't Hooft-Polyakov monopoles from pure SU(3) gauge theory

Ebert, D., Faustov, R.N. \& Galkin, V.O., Two-photon decay rates of heavy quarkonia in the relativistic quark model

Ebert, D., Faustov, R.N. \& Galkin, V.O., Hyperfine splitting and leptonic decay rates in heavy quarkonia

El Gebaly, H., see Ismail

Ellis, J., see Anselmo

Elster, C., see Fachruddin

Elster, Ch., see Kamada

Ercolessi, E., One and quasi-one dimensional spin systems

Ermisch, K., see Sekiguchi

Esposito, G., see Bimonte

Esterline, J., see Tornow
A18(2003)426

A18(2003)2517

A18(2003)1355

A18(2003)2681

A18(2003)661

A18(2003)661

A18(2003)2319

A18(2003)366

A18(2003) 120

A18(2003)913

A18(2003)2587

A18(2003)503

A18(2003)955

A18(2003)2873

A18(2003)601

A18(2003)1597

A18(2003) 57

A18(2003)699

A18(2003) 452

A18(2003) 124

A18(2003)2329

A18(2003) 327

A18(2003) 2311

A18(2003) 258
Eugenio, P., see Tamura

Eygi, Z.D. \& Turan, G., Standard model CP violation in $B \rightarrow X_{d} \ell^{+} \ell^{-}$decays

Fachruddin, I., Elster, C. \& Glöckle, W., The protondeuteron break-up process in a three-dimensional approach

Faessler, A., see Yan

Falcone, D., see Buccella

Fan, H., New minimum uncertainty states for thermo field dynamics

Fan, H., New application of thermo field dynamics in simplifying the calculation of Wigner functions

Fang, D.Q., see Zhang

Faraoni, V., see Cooperstock

Faustov, R.N., see Ebert

Faustov, R.N., see Ebert

Fazio, A.R., see Lemes

Fazio, A.R. \& Savvidy, G.K., Twoloop worldsheet effective action

Feng, J.-L., see Li

Feng, X.-C., see Li

Ferrara, S., see Andrianopoli

Fiasconaro, A., see Song

Filimonov, A.S., see Fortov

Firsova, N.E., $S$-matrices for spinor particles on ReissnerNordström black holes

Fix, A., see Arenhövel

Fleischer, R., Exploring CPviolation through $B$ decays

Foot, R., On super-Kamiokande's multi-ring analysis

Foot, R., Are four neutrino models ruled out?

Fortov, V.E., Filimonov, A.S., Gryaznov, V.K., Nikolaev, D.N., Ternovoi, V.Ya. \& Keeler, R.N., The generation of a non-ideal plasma by shock compression of high-porosity $\mathrm{SiO}_{2}$-aerogel

Frampton, P.H., Strongelectroweak unification at about $4 \mathrm{TeV}$

Franklin, G.B., see Tamura

Franz, J., see Tamura

Fujita, K., see Maeda
A18(2003)85

A18(2003)2735

A18(2003)452

A18(2003) 370

A18(2003)1819

A18(2003)677

A18(2003)733

A18(2003) 151

A18(2003)1037

A18(2003)601

A18(2003)1597

A18(2003)711

A18(2003)2817

A18(2003)2775

A18(2003)2775

A18(2003)1001

A18(2003)317

A18(2003)1835

A18(2003)1287

A18(2003)190

A18(2003)1413

A18(2003)2071

A18(2003)2079

A18(2003)1835

A18(2003)1377

A18(2003)85

A18(2003) 85

A18(2003)298 
Fujiwara, Y., Kohno, M., Suzuki, Y., Nakamoto, C., Miyagawa, K. \& Nemura, H., Quark-model interactions for the complete baryon octet and their applications to few-baryon systems

Fukuyama, T. \& Kikuchi, T., Renormalization group equation of quark-lepton mass matrices in the SO(10) model with two-Higgs scalars

Fukuyama, T., see Matsuda

Fülöp, T., Miyazaki, H. \& Tsutsui, I., Quantum force due to distinct boundary conditions

Funaki, Y., Horiuchi, H., Tohsaki, A., Schuck, P. \& Röpke, G., Description of ${ }^{8} \mathrm{Be}$ as deformed gas-like two-alpha-particle states

Furuichi, M., Shimizu, K. \& Takeuchi, S., Description of SU(3) $s$-wave and $p$-wave baryons

Gaitan, R., On consistence of material coupling in a $\operatorname{GL}(3, R)$ gauge formulation of gravity

Galajinsky, A.V., Remarks on $N=4$ superconformal extension of the Calogero model

Galkin, V.O., see Ebert

Galkin, V.O., see Ebert

Gan, L., see Tamura

Gangopadhyay, D., see Bhattacharyya

Gao, D.-N., see Jiang

Gao, H. (for Hall A, E94-104 and E02-010 Collabs.), Charged pion photoproduction from hydrogen and deuterium at Jefferson Lab

García-Alvarez, D., see Aguado

Geyer, H.B., see Jellal

Ghosh Choudhury, A., Khanra, B. \& Roy Chowdhury, A., Canonical Bäcklund transformation for a discrete integrable chain and its associated properties

Ghosh, D., Deb, A., Sahoo, S.R., Haldar, P.K. \& Bhattacharyya, S., Observation of void probability scaling of proton emission in high energy nucleus-nucleus collisions
A18(2003)102

A18(2003)719

A18(2003)1803

A18(2003)2863

A18(2003) 170

A18(2003)362

A18(2003)1753

A18(2003)1493

A18(2003)601

A18(2003)1597

A18(2003) 85

A18(2003)2207

A18(2003)977

A18(2003) 215

A18(2003)2303

A18(2003)927

A18(2003)1127

A18(2003)2281
Ghosh, S., Mandal, S. \& Chakrabarty, S., Impossibility of spin polarized states for neutron star/proto-neutron star matter in $\beta$-equilibrium condition

Giachetta, G., Mangiarotti, L. \& Sardanashvily, G., Noether conservation laws in higherdimensional Chern-Simons theory

Giacomelli, G. \& Margiotta, A., The MACRO experiment

Giansiracusa, G., see Song

Gibbs, W.R., The contribution of the light quark condensate to the $\pi N$ sigma term

A18(2003)1297

Giedt, J., Optical unification

Gilman, R., Neutron spin structure with polarized ${ }^{3} \mathrm{He}$ at Jefferson Lab Hall A

Gilman, R., Polarization measurements in $\pi^{0}$ photoproduction from the proton

Giunti, C., Last CPT-invariant hope for LSND neutrino oscillations

Glöckle, W., see Kamada

Glöckle, W., see Miyagawa

Glöckle, W., see Fachruddin

Gojuki, S. \& Oryu, S., Polarization effects in the ${ }^{3} \mathrm{He}(d, p){ }^{4} \mathrm{He}$ fusion reaction

Golak, J., see Kamada

Goldman, T., see Wang

Golowich, E., see Maltman

Goncharov, Yu.P. \& Choban, E.A., Dirac equation in the confining SU(3)-Yang-Mills field and the relativistic effects in quarkonia spectra

Gönül, O., see Özer

González Trotter, D.E., see Tornow

González-Díaz, P.F., Quintessential inflation without a potential

González-Robles, V.M., Bell's inequalities for any spin

Gorski, K.M., see Mukherjee

Govindarajan, T.R., Information from quantum blackhole physics

Gozzi, E., see Abrikosov, Jr., Greenfield, M.B., see Maeda
A18(2003)2645

A18(2003)2001

A18(2003)317

A18(2003)1171

A18(2003) 1625

A18(2003)272

A18(2003)286

A18(2003)1179

A18(2003)124

A18(2003)290

A18(2003)452

A18(2003)302

A18(2003) 124

A18(2003)356

A18(2003)366

A18(2003) 1661

A18(2003)2581

A18(2003) 258

A18(2003)2599

A18(2003)1931

A18(2003)1145

A18(2003)2337

A18(2003)2347

A18(2003)298 
Grothe, M., Mixing in the $D^{0}$ system - Results from collider experiments

Gryaznov, V.K., see Fortov

Gunzig, E., see Abramo

Guo, H.-Y., see Zhou

Guo, Z.Y., see Zhang

Gupta, K.S. \& Sen, S., Hidden degeneracy in the brick wall model of black holes

Gupta, K.S., Quantum inverse square interaction

Gupta, V., see Chaturvedi

Gupta, V., see Chaturvedi

Gurzadyan, V.G. \& Xue, S.-S., On the estimation of the current value of the cosmological constant

Gusso, A., de S. Pires, C.A. \& Rodrigues da Silva, P.S., Neutrino mixing and the minimal 3-3-1 model

Gutsche, T., see Yan

Haldar, P.K., see Ghosh

Haller, K. \& Ren, H.-C., The Coulomb interaction and the inverse Faddeev-Popov operator in QCD

Harada, K., see Yoshino

Harada, T., see Shinmura

Hashimoto, O., see Tamura

Hatanaka, K., see Maeda

Hatanaka, K., see Shimizu

Hatanaka, K., see Yagita

Hatanaka, K., see Tamii

Hatanaka, K., see Tamii

Hatano, M., see Saito

Hatano, M., see Maeda

Hatano, M., see Sekiguchi

Hatano, M., see Tamii

Hatano, M., see Tamii

Hawkins, E., Noncommutative rigidity for physicists

Hayakawa, H., Hosokawa, K. \& Kurimoto, T., $\left|V_{u b}\right|$ determination by $B \rightarrow D_{s} \pi$

Hayakawa, T., see Tamura

Hayashi, M.J., Watanabe, T., Aizawa, I. \& Aketo, I., Dilatonic inflation and SUSY breaking in string-inspired supergravity

Herrera, R., see Cárdenas

Hirooka, D., see Maeda

Hirooka, D., see Tamii
A18(2003)1

A18(2003) 1835

A18(2003) 1043

A18(2003)1911

A18(2003)151

A18(2003)1463

A18(2003)2355

A18(2003) 1635

A18(2003)1825

A18(2003)561

A18(2003)1849

A18(2003) 370

A18(2003)2281

A18(2003)2749

A18(2003)444

A18(2003) 128

A18(2003) 85

A18(2003) 298

A18(2003)313

A18(2003) 322

A18(2003)410

A18(2003)440

A18(2003)294

A18(2003)298

A18(2003) 327

A18(2003)410

A18(2003) 440

A18(2003)2363

A18(2003) 1557

A18(2003) 85

A18(2003) 2785

A18(2003)2039

A18(2003)298

A18(2003)410
Hiyama, E., Kamimura, M., Motoba, T., Yamada, T. \& Yamamoto, Y., Few-body aspects of hypernuclear physics

Horiuchi, H., see Funaki

Hosokawa, K., see Hayakawa

Hotchi, H., see Tamura

Hou, W.-S., see Chua

Howell, C.R., see Tornow

Howell, C.R., see Tornow

$\mathrm{Hu}$, Z.G., see Zhang

Hubeny, V.E. \& Rangamani, M., Horizons and plane waves: A review

Hurtado, J.Q., Inflationary dilatonic de Sitter universe from $N=4$ super-Yang-Mills theory perturbed by scalars

Hutcheson, A., see Tornow

Hwang, W.-Y.P., Early-universe phase transitions and YangMills theories

Iglesias, A., see Corradini

Ikeda, K., see Myo

Ikeda, K., see Ito

Ikeda, K., see Masui

Ikeda, T., see Maeda

Iltan, E.O., Neutral Higgs $H^{0} \rightarrow$ $h^{0}\left(A^{0}\right) l_{\mathrm{i}}^{-} l_{j}^{+}$decay

Imai, K., see Tamura

Inoue, T., see Sasaki

Iovane, G., see Capozziello

Ishida, T., see Yagita

Ishida, T., Yagita, T., Ochi, S., Nozoe, S., Tsuruta, K., Nakamura, F., Schieck, H.G.P.G. \& Sagara, K., Search for space star anomaly in pd breakup reaction at $13 \mathrm{MeV}$

Ishida, T., see Tamii

Ishii, N., see Mineo

Isidro, J.M., Duality, quantum mechanics and (almost) complex manifolds

Islam, M.M., Luddy, R.J. \& Prokudin, A.V., $p p$ elastic scattering at LHC and nucleon structure

Ismail, M., Osman, M.M., El Gebaly, H. \& Abou-Shady, H., Orientation and deformation dependence of the reaction cross-section for a deformed target nucleus

Isupov, A.Yu., see Saito
A18(2003)95

A18(2003) 170

A18(2003) 1557

A18(2003) 85

A18(2003) 1763

A18(2003) 258

A18(2003)282

A18(2003)151

A18(2003)2699

A18(2003)1257

A18(2003)282

A18(2003)669

A18(2003)1343

A18(2003)166

A18(2003) 178

A18(2003)186

A18(2003)298

A18(2003)1539

A18(2003)85

A18(2003)131

A18(2003)905

A18(2003)322

A18(2003)436

A18(2003)440

A18(2003)384

A18(2003)1975

A18(2003)743

A18(2003) 57

A18(2003)294 
Ito, M., Katō, K. \& Ikeda, K., New cluster model based on the generator coordinate method

Itoh, K., see Maeda

Itonaga, K., Motoba, T. \& Ueda, $\mathrm{T}$., Nonmesonic weak decays of single- $\Lambda$ and double- $\Lambda$ hypernuclei of light mass systems

Jellal, A. \& Geyer, H.B., Second virial coefficient for noncommutative space

Jellal, A., Noncommutativity parameter and composite fermions

Jena, P.K. \& Singh, L.P., SU(2) chiral sigma model study of phase transition in hybrid stars

Jia, C.-S., Yi, L.-Z., Sun, Y., Liu, J.-Y. \& Sun, L.-T., New solvable pseudo-Hermitian potential models with real spectra

Jiang, J.-H., Gao, D.-N. \& Yan, M.-L., $K_{L} \rightarrow \gamma v \bar{v}$ decay beyond the standard model

Jing, S., see Li

Joglekar, S.D., Additional considerations in the definition and renormalizationn of noncovariant gauges

Joy, M. \& Kuriakose, V.C., Phase transitions for a $\phi^{6}$ model in $(2+1)$-dimensional curved spacetime

Julia-Diaz, B. \& Lee, T.-S.H., Quark-exchange mechanism of $\gamma d \rightarrow n p$ reaction at $2-6 \mathrm{GeV}$

Kakushadze, Z., see Corradini

Kalantar-Nayestanaki, N., see Sekiguchi

Kamada, H., Glöckle, W., Golak, J. \& Elster, Ch., Lorentz boosted NN potential for fewbody systems: Application to the three-nucleon bound state

Kamada, H., see Maeda

Kamalov, S.S., see Yang

Kamani, D., Noncommutativity and its freedoms due to the $p p$-wave and background gauge fields

Kamani, D., Noncommutativity of the moving D2-brane worldvolume

Kamimura, M., see Hiyama
A18(2003) 178

A18(2003)298

A18(2003)135

A18(2003)927

A18(2003)1473

A18(2003)2135

A18(2003)1247

A18(2003) 977

A18(2003)1523

A18(2003)843

A18(2003)937

A18(2003) 200

A18(2003)1343

A18(2003)327

A18(2003)124

A18(2003) 298

A18(2003) 248

A18(2003)2125

A18(2003)2195

A18(2003) 95
Kamimura, M., see Kino

A18(2003)388

A18(2003)298

A18(2003)313

A18(2003) 322

A18(2003)410

A18(2003)440

Kamiya, J., see Tamii

Kar, S., D-branes, cyclic symmetry and noncommutative geometry

Karagöz, N., see Boz

Karatheodoris, G., Pinzul, A. \& Stern, A., Fate of the BornInfeld solution in string theory

Kato, H., see Saito

Kato, H., see Maeda

Kato, H., see Sekiguchi

Kato, H., see Tamii

Katō, K., see Kurokawa

Katō, K., see Myo

Katō, K., see Ito

Katō, K., see Masui

Kawamura, H., Kodaira, J., Qiao, C.-F. \& Tanaka, K., Transverse momentum distribution in the $B$ mesons in the heavy-quark limit: The Wandzura-Wilczek part

Keeler, R.N., see Fortov

Kelley, J.H., see Tornow

Kerman, A.J., see Chin

Ketov, S.V., More on the gaugefixed D3-brane action with dilaton-axion coupling from $N=1$ superspace

Khanra, B., see Ghosh Choudhury Khaustov, P., see Tamura

Kikuchi, T., see Fukuyama

Kim, S.-K., see Kim

Kim, Y-W., Kim, S.-K. \& Park, Y.-J., BFT Hamiltonian embedding of massive theory with one- and two-form gauge fields

Kino, Y., Kudo, H. \& Kamimura, M., High-precision Coulomb three-body calculation of antiprotonic helium atoms

Kiselev, V.V., A gluon condensate term in a heavy quark mass

Kishimoto, T., see Tamura

Kitamura, Y., see Maeda

Kitamura, Y., see Tamii

Klapdor-Kleingrothaus, H.V. \& Sarkar, U., Consequences of neutrinoless double beta decay and WMAP
A18(2003)1053

A18(2003) 1601

A18(2003) 1681

A18(2003)294

A18(2003)298

A18(2003) 327

A18(2003)410

A18(2003) 162

A18(2003) 166

A18(2003) 178

A18(2003)186

A18(2003)799

A18(2003) 1835

A18(2003)282

A18(2003)398

A18(2003) 1887

A18(2003)1127

A18(2003) 85

A18(2003)719

A18(2003)2287

A18(2003)2287

A18(2003)388

A18(2003) 1735

A18(2003) 85

A18(2003)298

A18(2003)440

A18(2003)2243 
Klein, M., On supersymmetry breaking in intersecting brane models

Kobdaj, C., see Yan

Kodaira, J., see Kawamura

Kohno, M., see Fujiwara

Koike, Y., Nuclear three-body resonances

Kokubo, Y., see Deguchi

Körner, J.G. \& Liu, C., A supersymmetric model with the gauge symmetry $\mathrm{SU}(3)_{1} \times$ $\mathrm{SU}(2)_{1} \times \mathrm{U}(1)_{1} \times \mathrm{SU}(3)_{2} \times$ $\mathrm{SU}(2)_{2} \times \mathrm{U}(1)_{2}$

Kou, S.-P., The mechanism of Migdal recursion relation and phase diagrams of Yang-Mills fields

Kowalski-Glikman, J., see Błaut

Krajewski, T., see Dąbrowski

Krause, A., On the flattening of negative curvature via $T$-duality with a non-constant $B$-field

Krein, G., see Battistel

Kreyerhoff, G., see Barshay

Kreyerhoff, G., see Barshay

Kuboki, H., see Maeda

Kuboki, H., see Tamii

Kudo, H., see Kino

Kumasaka, H., see Saito

Kuriakose, V.C., see Joy

Kurimoto, T., see Hayakawa

Kurokawa, C. \& Kato, K., Threealpha structures and alpha-condensation in ${ }^{12} \mathrm{C}$

Ladygin, V.P., see Saito

Ladygin, V.P., see Shimizu

Ladygina, N.B., see Saito

Lamanna, G., Astrophysics and particle physics in space with the alpha magnetic spectrometer

Lambiase, G., Cerenkov's effect and neutrino oscillations in loop quantum gravity

Lambiase, G., see Capozziello

Lambiase, G., MSW effect in loop quantum gravity and constraints on parameters from neutrino-antineutrino transitions
A18(2003)2545

A18(2003) 370

A18(2003) 799

A18(2003)102

A18(2003) 418

A18(2003)2051

A18(2003)967

A18(2003)2653

A18(2003) 1711

A18(2003)2371

A18(2003)2571

A18(2003)2255

A18(2003)477, 875(E)

A18(2003)2887

A18(2003)298

A18(2003)440

A18(2003) 388

A18(2003)294

A18(2003)937

A18(2003)1557

A18(2003) 162

A18(2003)294

A18(2003) 313

A18(2003)294

A18(2003)1951

A18(2003) 23

A18(2003)905

A18(2003)1397
Lan, D.M., Quantum potential applied in the coupling of classical gravity and quantum mechanics

A18(2003) 2563

A18(2003)2371

A18(2003)200

A18(2003)347

A18(2003)609

Leiva, C., see Amézaga

Lemes, V.E.R., Sarandy, M.S., Sorella, S.P., Picariello, M. \& Fazio, A.R., Ghost condensates in Yang-Mills theories in nonlinear gauges

Lévai, G., see Williams

Li, D.-M., Ma, B., Yu, H., Yao, Q.K., Feng, J.-L. \& Feng, X.-C., Meson mixing and the mass of the isodoublet of $2{ }^{1} S_{0}$ nonet

Li, J.F., see Zhang

Li, J.R., see Meng

Li, J.S., see Liu

Li, J.X., see Zhang

Li, N., see Ren

Li, Q. \& Zhao, E., The role of isovector meson exchanges to nucleon-nucleon elastic cross-section

Li, W.F., see Zhang

Li, X.-Y. \& Ma, E., Gauge model of quark-lepton nonuniversality

Li, Y. \& Jing, S., Charged coherent states related to $\mathrm{su}_{q}(2)$ covariance

Lima-Santos, A., see Abdalla

Litvinenko, V.N., see Tornow

Liu, B., see Long

Liu, C., see Körner

Liu, F., see Cheng

Liu, J.-Y., see Jia

Liu, L.J., see Yue

Liu, Y.X., Li, J.S. \& Bao, C.G., Low-lying dibaryon states in a symmetry analysis

Lizzi, F., Vitale, P. \& Zampini, A., From the fuzzy disc to edge currents in Chern-Simons theory

Lledó, M.A., see Andrianopoli

Lombardo, U., see Song

Long, Z.-W. \& Liu, B., A new approach treating constrained systems based on the consistency condition of constraints
A18(2003)711

A18(2003)1901

A18(2003)2775

A18(2003)151

A18(2003)2029

A18(2003)414

A18(2003) 151

A18(2003)174

A18(2003) 2713

A18(2003)151

A18(2003)1367

A18(2003) 1523

A18(2003)1435

A18(2003)282

A18(2003)2273

A18(2003)967

A18(2003)1107

A18(2003) 1247

A18(2003)2187

A18(2003)414

A18(2003)2381

A18(2003)1001

A18(2003)317

A18(2003)2273 
Love, S.T., Gauging internal fermionic symmetries and spin$3 / 2$ fields

Lu, X.-F., see Sun

Lucha, W. \& Schöberl, F.F., The charmed strange meson system

Luddy, R.J., see Islam

Lukierski, J., Lyakhovsky, V.D. \& Mozrzymas, M., к-deformations of $D=3$ conformal versus deformations of $D=4$ AdS symmetries

Lukierski, J., see Borowiec

Lyakhovsky, V.D., see Lukierski

Lynden-Bell, R.M., see Zhang

Ma, B., see $\mathrm{Li}$

Ma, E., see Li

Ma, G.L., see Zhang

Ma, Y.G., see Zhang

Macri, R.A., see Tornow

Maeda, Y., see Saito

Maeda, Y., Sakia, H., Tamii, A., Sakoda, S., Kato, H., Hatano, M., Saito, T., Uchigashima, N., Kuboki, H., Hatanaka, K., Hirooka, D., Shimizu, Y., Kitamura, Y., Fujita, K., Sakamoto, N., Okamura, H., Suda, K., Ikeda, T., Itoh, K., Sakemi, Y., Wakasa, T., Kamiya, J., Yako, K., Sekiguchi, K., Sato, Y., Greenfield, M.B., Rapaport, J. \& Kamada, H., Measurement of differential cross sections and vector analyzing powers for the $\vec{n} d$ reaction at $250 \mathrm{MeV}$

Maeda, Y., see Shimizu

Maeda, Y., see Sekiguchi

Maeda, Y., see Tamii

Maeda, Y., see Tamii

Majumdar, D., Relic densities for Kaluza-Klein dark matter

Malakhov, A.I., see Saito

Malik, R.P., Superfield approach to a novel symmetry for nonAbelian gauge theory

Malik, R.P., Hamiltonian and Lagrangian dynamics in a noncommutative space

Maltman, K., Cirigliano, V., Donoghue, J.F. \& Golowich, E., The chiral limit $K \rightarrow \pi \pi$ matrix elements of the electroweak penguin operators $Q_{7,8}$
A18(2003)1099

A18(2003)1485

A18(2003)2837

A18(2003)743

A18(2003)753

A18(2003)1157

A18(2003) 753

A18(2003)406

A18(2003)2775

A18(2003)1367

A18(2003) 151

A18(2003) 151

A18(2003) 258

A18(2003)294

A18(2003) 298

A18(2003)313

A18(2003) 327

A18(2003)410

A18(2003)440

A18(2003)1705

A18(2003)294

A18(2003)2147(E)

A18(2003)2795

A18(2003)366
Mandal, S., see Ghosh

Mangano, G., Miele, G. \& Pettorino, V., Coupled quintessence and the coincidence problem

Mangiarotti, L., see Giachetta

Mansfield, P., see Davies

Manvelyan, R., On marginal deformation of WZNW model and $P P$-wave limit of deformed $\mathrm{AdS}_{3} \times S^{3}$ string geometry

Margiotta, A., see Giacomelli

Markoff, D.M., see Tornow

Marmo, G., see Bimonte

Mart, T., see Miyagawa

Martin, X., Simulating the scalar field using the fuzzy sphere

Masuda, T., see Aoyama

Masui, H., Myo, T., Katō, K. \& Ikeda, K., Coupled-channel study for O-isotopes with the core plus valence neutrons model

Matsuda, K., Fukuyama, T. \& Nishiura, H., Can the Majorana neutrino $\mathrm{CP}$-violating phases be restricted?

Matsuda, M., see Yoshino

Matsuda, M., see Nagata

Mauro, D., see Abrikosov, Jr.

May, M., see Tamura

Maziashvili, M., Particle production related to the tunneling in false vacuum decay

Maziashvili, M., Proper fluctuations associated with quantum tunneling in field theory

Mazzacurati, L., see Casadio

McKeown, R.D., The nucleon's mirror image: Revealing the strange and unexpected

Meggs, W.J., The physics of biology

Melnitchouk, W., see Detmold

Meng, J., Chen, J.S. \& Li, J.R., The L-G phase transition and the nucleon-nucleon interaction potential within QHD-I model

Meyer, A., The CDF experiment at the Tevatron: The first two years of run II

Micu, L., Sets of analytical solutions of the Dirac equation with confining potentials
A18(2003)831

A18(2003)1297

A18(2003)2645

A18(2003) 1517

A18(2003) 1531

A18(2003)2001

A18(2003)258

A18(2003)2311

A18(2003)290

A18(2003)2389

A18(2003)553

A18(2003)186

A18(2003) 1803

A18(2003)444

A18(2003)448

A18(2003) 2347

A18(2003)85

A18(2003)993

A18(2003)1895

A18(2003)651

A18(2003) 75

A18(2003)2397

A18(2003)2681

A18(2003)2029

A18(2003)1643

A18(2003)2895 
Miele, G., see Mangano

Mignemi, S., Two-dimensional gravity with an invariant energy scale and arbitrary dilaton potential

Mikhailov, S., see Tornow

Minami, S., see Tamura

Minami, S., see Yagita

Mineo, H., Bentz, W., Thomas, A.W., Ishii, N. \& Yazaki, K., Structure function in nuclear matter in the NJL model

Mirea, M., Landau-Zener effect in superfluid nuclear systems

Miura, Y., see Tamura

Miyagawa, K., see Fujiwara

Miyagawa, K., Mart, T., Bennhold, C. \& Glöckle, W., Electromagnetic $K^{+}$production on light nuclei with beam and hyperon recoil polarizations

Miyazaki, H., see Fülöp

Miyoshi, T., see Tamura

Mizunuma, K., see Tamura

Mkrtchyan, H. \& Mkrtchyan, R., Little groups of Preon branes

Mkrtchyan, R., see Mkrtchyan

Modanese, G., Local contribution of a quantum condensate to the vacuum energy density

Molodtsov, S.V. \& Zinovjev, G.M., Role of quark interactions with instanton liquid in colour superconductivity phase

Monte, E.M., see Dahia

Morando, P. \& Tarallo, M., HyperHamiltonian dynamics and quaternionic regularity

Motoba, T., see Hiyama

Motoba, T., see Itonaga

Mozrzymas, M., see Lukierski

Mukherjee, P., Souradeep, T., Ratra, B., Sugiyama, N. \& Gorski, K.M., OVRO CMB anisotropy measurement constraints on flat- $\Lambda$ and open CDM cosmogonies

Mukhopadhyay, B., see Singh

Mukunda, N., Generalized coherent states and operator descriptions in quantum mechanics

Muslih, S.I., The canonical path integral quantization of chiral Schwinger model

Myint, K.S., see Shinmura
A18(2003)831

A18(2003)643

A18(2003) 282

A18(2003) 85

A18(2003)322

A18(2003)384

A18(2003)1809

A18(2003) 85

A18(2003)102

A18(2003) 290

A18(2003)2863

A18(2003) 85

A18(2003)85

A18(2003)2665

A18(2003)2665

A18(2003)683

A18(2003) 817

A18(2003)1773

A18(2003)1841

A18(2003)95

A18(2003)135

A18(2003) 753

A18(2003)1145

A18(2003)779

A18(2003)2405

A18(2003) 1187

A18(2003) 128
Myo, T., Katō, K., Aoyama, S. \& Ikeda, K., Resonances and continuums in the three-body breakup of halo nuclei with the complex scaling method

Myo, T., see Masui

Myung, Y.S., Absorption crosssection in de Sitter space

Nagae, T., see Tamura

Nagata, J., see Yoshino

Nagata, J., Yoshino, H. \& Matsuda, M., Phase-shift analyses of elastic $p p$ scattering in cosy region

Nair, V.P., Yang-Mills theory, $2+1$ and $3+1$ dimensions

Nakamoto, C., see Fujiwara

Nakamura, F., see Ishida

Nakamura, S.N., see Tamura

Nakano, T., Laser-electron photon project at SPing-8

Nakazawa, K., see Tamura

Nakazawa, K., Systematic study of double strrangeness system by emulsion-counter hybrid method

Nanopoulos, D.V., see Anselmo

Narayan, M. \& Sankar, S.U., Analytical calculation of matter effects in two mass-scale neutrino oscillations

Navarro, I., Phenomenology of low-scale supersymmetry breaking models

Nawafleh, K.I., see Rabei

Ndimubandi, J., see Debergh

Neidel, E.M., see Tornow

Nemura, H., see Fujiwara

Nemura, H., Akaishi, Y. \& Suzuki, Y., Ab initio calculation of ${ }_{\Lambda}^{5} \mathrm{He}$ with explicit $\Sigma$ admixture

Ng, Y.J., Selected topics in Planckscale physics

Nikolaev, D.N., see Fortov

Nishikawa, J., see Saito

Nishikawa, J., see Sekiguchi

Nishikawa, M., On singular potential of the Schrödinger equation

Nishiura, H., see Matsuda

Noro, T., see Shimizu

Noro, T., see Yagita

Nozoe, S., see Ishida

O'Connor, D., Field theory on low dimensional fuzzy spaces

Ochi, S., see Ishida
A18(2003) 166

A18(2003)186

A18(2003)617

A18(2003) 85

A18(2003)444

A18(2003)448

A18(2003)2415

A18(2003) 102

A18(2003)436

A18(2003) 85

A18(2003)208

A18(2003) 85

A18(2003)116

A18(2003)699

A18(2003)569

A18(2003)2227

A18(2003)1591

A18(2003)1013

A18(2003) 258

A18(2003)102

A18(2003)139

A18(2003) 1073

A18(2003)1835

A18(2003)294

A18(2003)327

A18(2003)1991

A18(2003)1803

A18(2003)313

A18(2003)322

A18(2003)436

A18(2003)2423

A18(2003)436 
Oda, I., Covariant matrix model of superparticle in the pure spinor formalism

Oelsner, M., see Yuan

Ohnishi, T., see Saito

Ohnishi, T., see Sekiguchi

Oka, M., see Sasaki

Okamura, H., see Saito

Okamura, H., see Maeda

Okamura, H., see Sekiguchi

Okamura, H., see Tamii

Okayasu, Y., see Tamura

Omari, H.Y.R., see Rabei

Ookouchi, Y. \& Watabiki, Y., Effective superpotentials for $\mathrm{SO} / \mathrm{Sp}$ with flavor from matrix models

Oryu, S., see Watanabe

Oryu, S., see Gojuki

Osman, M.M., see Ismail

Özer, O. \& Gönül, O., New exact treatment of the perturbed Coulomb interactions

Padmanabhan, T., Topological interpretation of the horizon temperature

Palmer, J.S., see Tsou

Pan, J., see Zhou

Pang, H.-R., see Wang

Park, Y.-J., see Kim

Patiño, L. \& Quevedo, H., Bosonic and fermionic behavior in gravitational configurations

Pauchy Hwang, W.-Y., Nucleon properties in the expanding universe

Pavlov, A. \& Pavlova, Y., Evolution of elliptical galaxies and mechanism of formation of spiral galaxies

Pavlova, Y., see Pavlov

Pedroni, R.S., see Tornow

Pérez-Lorenzana, A. \& de S. Pires, C.A., Electric charge quantization and extra dimensions

Perna, R., The interaction between gamma-ray bursts and their environment

Pettorino, V., see Mangano

Piao, Y.-S., Zhang, X. \& Zhang, Y.-Z., Brane inflation from rotation of D4 brane

Picariello, M., see Lemes

Pile, P.H., see Tamura
A18(2003)1023

A18(2003)244

A18(2003)294

A18(2003) 327

A18(2003)131

A18(2003)294

A18(2003)298

A18(2003) 327

A18(2003) 440

A18(2003) 85

A18(2003)1591

A18(2003)1113

A18(2003) 182

A18(2003)302

A18(2003)57

A18(2003)2581

A18(2003)2903

A18(2003)807

A18(2003)1911

A18(2003)356

A18(2003)2287

A18(2003)1331

A18(2003)374

A18(2003)2265

A18(2003)2265

A18(2003)258

A18(2003)65

A18(2003)2611

A18(2003)831

A18(2003)2109

A18(2003) 711

A18(2003)85
Pinayev, I.V., see Tornow

Ping, J.-L., see Wang

Pinzul, A. \& Stern, A., $W_{\infty}$ algebras from noncommutative Chern-Simons theory

Pinzul, A., see Karatheodoris

Pinzul, A. \& Stern, A., Edge states from defects on the noncommutative plane

Pollock, M.D., On the relaxation of superstring axion miniclusters

Porrati, M., Higgs phenomenon for the graviton in AdS space

Prešnajder, P., Gauge fields on the fuzzy sphere

Prokudin, A.V., see Islam

Qiao, C.-F., see Kawamura

Quesne, C., Fractional supersymmetric quantum mechanics, topological invariants and generalized deformed oscillator algebras

Quevedo, H., see Patiño

Quinn, B.P., see Tamura

Rabei, E.M., Nawafleh, K.I., Abdelrahman, Y.S. \& Omari, H.Y.R., Hamilton-Jacobi treatment of Lagrangians with linear velocities

Rahaman, F., Superconducting cosmic string in Lyra geometry

Rajeev, S.G., A theory of errors in quantum measurement

Rama, S.K., Classical velocity in к-deformed Poincaré algebra and a maximum acceleration

Ramón Medrano, M. \& Sanchez, N.G., New dual relations between quantum field theory and string regimes in curved backgrounds

Ramond, P., Adventures in eleven dimensions

Randjbar-Daemi, S., Abrikozov vortex and branes

Rangamani, M., see Hubeny

Rapaport, J., see Maeda

Ratra, B., see Mukherjee

Ravindran, V., Smith, J. \& van Neerven, W.L., Differential cross-sections for Higgs production

Ray, S., see Sinha
A18(2003)282

A18(2003)356

A18(2003)1215

A18(2003)1681

A18(2003)2509

A18(2003)947

A18(2003)1793

A18(2003)2431

A18(2003)743

A18(2003)799

A18(2003)515

A18(2003)1331

A18(2003) 85

A18(2003)1591

A18(2003)41

A18(2003)2439

A18(2003)527

A18(2003)2537

A18(2003)2451

A18(2003)2459

A18(2003)2699

A18(2003)298

A18(2003)1145

A18(2003)1721

A18(2003)661 
Rebouças, M.J. \& Santos, J., A note on Segre types of secondorder symmetric tensors in 5-D brane-world cosmology

Ren, H.-C., see Haller

Ren, Z.Z., see Zhang

Ren, Z.Z., Li, N., Zhang, H.Y. \& Shen, W.Q., A three-body model on neutron halo in exotic light nuclei

Reznikov, S.G., see Saito

Rizov, V.A., see Yazadjiev

Rodgers, V.G.J. \& Yasuda, T., From diffeomorphisms to dark energy?

Rodrigues da Silva, P.S., see Gusso

Rojas, M., see Carrion

Rojas, M., see Carrion

Romero, C., see Dahia

Romero, C., see Barros

Romero, C., see Barros

Romero, J.M. \& Vergara, J.D., The Kepler problem and noncommutativity

Röpke, G., see Funaki

Rosu, H.C., Quantum Hamiltonians and prime numbers

Roy Chowdhury, A., see Ghosh Choudhury

Roy, D.P., see Desai

Royon, C., Diffractive Higgs boson production at Tevatron and LHC: An experimental review

Rusek, A., see Tamura

Saa, A., see Abramo

Safonova, M., see Turakulov

Sagara, K., see Shimizu

Sagara, K., see Yagita

Sagara, K., see Ishida

Saha, A. (for the Jefferson Lab Hall A Collab.), Detailed study of the few nucleon systems at Jefferson Lab

Sahoo, B.K. \& Singh, L.P., Cosmic evolution in generalised Brans-Dicke theory

Sahoo, S.R., see Ghosh

Saito, T., Hatano, M., Kato, H., Maeda, Y., Sakai, H., Sakoda, S., Tamii, A., Uchigashima, N., Ladygin, V.P., Isupov, A.Yu., Ladygina, N.B., Malakhov,
A18(2003)2807

A18(2003)2749

A18(2003) 151

A18(2003) 174

A18(2003)294

A18(2003)2555

A18(2003)2467

A18(2003)1849

A18(2003)629

A18(2003) 787

A18(2003) 1773

A18(2003) 2117

A18(2003)2673

A18(2003)1673

A18(2003) 170

A18(2003)1205

A18(2003)1127

A18(2003)1355

A18(2003)2169

A18(2003)85

A18(2003)1043

A18(2003) 579

A18(2003) 313

A18(2003) 322

A18(2003)436

A18(2003)235

A18(2003)2725

A18(2003)2281
A.I., Reznikov, S.G., Uesaka, T., Yako, K., Ohnishi, T., Sakamoto, N., Sekiguchi, K., Kumasaka, H., Nishikawa, J., Okamura, H., Suda, K. \& Suzuki, R., Measurement of the analyzing powers for the $\vec{d} d \rightarrow{ }^{3} \mathrm{He} n$ and $\vec{d} d \rightarrow$ ${ }^{3} \mathrm{H} p$ reactions at intermediate energies

Saito, T., see Maeda

Saito, T., see Shimizu

Saito, T., see Sekiguchi

Saito, T., see Tamii

Saito, T., see Tamii

Sakai, H., see Saito

Sakai, H., Nd scattering at intermediate energy and threenucleon force effects

Sakai, H., see Shimizu

Sakai, H., see Sekiguchi

Sakai, H., see Tamii

Sakai, H., see Tamii

Sakamoto, N., see Saito

Sakamoto, N., see Maeda

Sakamoto, N., see Sekiguchi

Sakemi, Y., see Maeda

Sakemi, Y., see Shimizu

Sakia, H., see Maeda

Sakoda, S., see Saito

Sakoda, S., see Maeda

Sakoda, S., see Sekiguchi

Sakoda, S., see Tamii

Salisbury, D., Gauge fixing and observables in general relativity

Sami, M., Implementing power law inflation with tachyon rolling on the brane

Sanchez, N.G., see Ramón Medrano

Sanders, R.H., Missing mass as evidence for modified Newtonian dynamics at low accelerations

Sankar, S.U., see Narayan

Santiago, D.I., see Barbieri

Santos. J., see Rebouças

Sarandy, M.S., see Lemes

Sardanashvily, G., see Giachetta

Sarkar, U., see KlapdorKleingrothaus
A18(2003)294

A18(2003)298

A18(2003) 313

A18(2003)327

A18(2003)410

A18(2003)440

A18(2003)294

A18(2003)306

A18(2003)313

A18(2003) 327

A18(2003)410

A18(2003)440

A18(2003)294

A18(2003)298

A18(2003)327

A18(2003)298

A18(2003)313

A18(2003)298

A18(2003)294

A18(2003)298

A18(2003)327

A18(2003)410

A18(2003)2475

A18(2003)691

A18(2003)2537

A18(2003)1861

A18(2003)569

A18(2003) 2767

A18(2003)2807

A18(2003)711

A18(2003)2645

A18(2003)2243 
Sasaki, K., Inoue, T. \& Oka, M., Nonmesonic decay of $\Lambda$ and $\Lambda \Lambda$ hypernuclei

Sasao, J., see Tamura

Sato, Y., see Tamura

Sato, Y., see Maeda

Satou, Y., see Sekiguchi

Satou, Y., see Tamii

Sauer, P.U., see Yuan

Sauer, P.U., see Deltuva

Savvidy, G.K., see Fazio

Sawado, N., see Watanabe

Sawafta, R., see Tamura

Schieck, H.G.P.G., see Ishida

Schmidt, M. G. \& Stamatescu, I.-O., Matter determinants in background fields using random walk world line loops on the lattice

Schmitt, H., see Tamura

Schöberl, F.F., see Lucha

Schörner-Sadenius, T., The trigger of the Atlas experiment

Schuck, P., see Funaki

Schulze-Halberg, A., see Bose

Schulze-Halberg, A., Equivalence of time-dependent Schrödinger equations with constant and time-dependent mass

Schwamb, M., see Arenhövel

Sciolla, G., Recent measurements of CP-violation at the $B$-factories

Segar, J., see Aizawa

Sekiguchi, K., see Saito

Sekiguchi, K., see Maeda

Sekiguchi, K., see Shimizu

Sekiguchi, K., Sakai, H., Okamura, H., Tamii, A., Uesaka, T., Suda, K., Sakamoto, N., Wakasa, T., Satou, Y., Ohnishi, T., Yako, K., Sakoda, S., Kato, H., Maeda, Y., Hatano, M., Nishikawa, J., Saito, T., Uchigashima, N., Kalantar-Nayestanaki, N. \& Ermisch, K., Polarization transfer measurement for $d-p$ elastic scattering: To search for three nucleon force effects

Sekiguchi, K., see Tamii

Sekiguchi, K., see Tamii

Sen, S., see Gupta

Sen, S., see Tuite

Seth, K.K., Hybrids and glueballs A status report
A18(2003) 131

A18(2003) 85

A18(2003) 85

A18(2003) 298

A18(2003)327

A18(2003)440

A18(2003)244

A18(2003) 426

A18(2003)2817

A18(2003) 182

A18(2003) 85

A18(2003)436

A18(2003)1499

A18(2003) 85

A18(2003)2837

A18(2003)2149

A18(2003) 170

A18(2003)853

A18(2003)2829

A18(2003)190

A18(2003)2083

A18(2003) 885

A18(2003)294

A18(2003)298

A18(2003)313

A18(2003)327

A18(2003)410

A18(2003)440

A18(2003) 1463

A18(2003)2483

A18(2003)330
Sever, R. \& Aydemir, A., Family nonuniversal $Z^{\prime}$ and $b \rightarrow \mathrm{s} \gamma$ decay

Shen, P.-N., see Sun

Shen, W.Q., see Zhang

Shen, W.Q., see Ren

Shen, X.Y. (for the BES Collab.), Recent results from BES $J / \psi$ data

Shimizu, K., see Takeuchi

Shimizu, K., see Furuichi

Shimizu, Y., see Maeda

Shimizu, Y., Hatanaka, K., Sakemi, Y., Wakasa, T., Yoshida, H.P., Kamiya, J., Saito, T., Sakai, H., Tamii, A., Sekiguchi, K., Yako, K., Maeda, Y., Noro, T., Sagara, K. \& Ladygin, V.P., Three-body effects in $\bar{p} d$ elastic scattering at $250 \mathrm{MeV}$

A18(2003) 313

Shimizu, Y., see Tamii

Shinmura, S., Akaishi, Y., Myint, K.S. \& Harada, T., Hyperons in high-density nuclear medium: Is $\Lambda-\Sigma^{0}$ mixing large or small?

Shinohara, T., Renormalizable Abelian-projected effective gauge theory derived from quantum chromodynamics II

Singh, L.P., see Jena

Singh, L.P., see Sahoo

Singh, P. \& Dadhich, N., Localization of gravity in brane world cosmologies

A18(2003)545

A18(2003)1485

A18(2003) 151

A18(2003)174

A18(2003)340

A18(2003) 147

A18(2003)362

A18(2003)298

A18(2003)440

A18(2003)128

Singh, P. \& Mukhopadhyay, B., Gravitationally induced neutrino asymmetry

Singleton, D., see Dzhunushaliev Singleton, D., see Dzhunushaliev Sinha, M., Dey, J., Dey, M., Ray, S. \& Bhowmick, S., Have we observed the skin vibration of realistic strange stars (ReSS)?

Şirvanl1, B. \& Turan, G., Rare $B_{s} \rightarrow \gamma \nu v$ decay with polarized photon and new physics effects

Smith, J., see Ravindran

Soffer, J., see Bourrely

Song, H.Q., Baldo, M., Fiasconaro, A., Giansiracusa, G. \& Lombardo, U., Three nucleon correlations in nuclear medium

Song, X.-C., see Tian

Sorella, S.P., see Lemes
A18(2003)1403

A18(2003) 2135

A18(2003) 2725

A18(2003)983

A18(2003)779

A18(2003)955

A18(2003)2873

A18(2003)661

A18(2003)47

A18(2003)1721

A18(2003)771

A18(2003)317

A18(2003)1691

A18(2003)711 
Sorkin, R.D., Indecomposable ideals in incidence algebras Souradeep, T., see Mukherjee Sparano, G., Superintegrable systems and recursion operators

Stamatescu, I.-O., see Schmidt

Stenkin, Yu.V., Does the "knee" in primary cosmic ray spectrum exist?

Stern, A., see Pinzul Stern, A., see Karatheodoris

Stern, A., see Pinzul

Stoenescu, G., see Calian

Stornaiolo, C., see Bimonte

Su, R.-K., see Wang

Su, R.-K., see Zhang

Suda, K., see Saito

Suda, K., see Maeda

Suda, K., see Sekiguchi

Suda, K., see Tamii

Suebka, P., $\bar{N} N$ atoms in Paris potential

Sugiyama, N., see Mukherjee

Sultansoy, S., see Ciftci

Sun, B.-X., Lu, X.-F., Shen, P.-N. \& Zhao, E.-G., Effective photon mass in nuclear matter and finite nuclei

Sun, L.-T., see Jia

Sun, Y., see Jia

Sun, Z.Y., see Zhang

Sutter, R., see Tamura

Suzuki, R., see Saito

Suzuki, Y., see Fujiwara

Suzuki, Y., see Nemura

Takahashi, H., see Tamura

Takeuchi, S. \& Shimizu, K., Nonlocality in the quark-model induced two-baryon potential

Takeuchi, S., see Furuichi

Tamii, A., see Saito

Tamii, A., see Maeda

Tamii, A., see Shimizu

Tamii, A., see Sekiguchi

Tamii, A., Hatano, M., Kato, H., Maeda, Y., Saito, T., Sakai, H. Sakoda, S., Uchigashima, N., Hatanaka, K., Hirooka, D., Kamiya, J., Wakasa, T., Yako, K., Sekiguchi, K. \& Uesaka, T., Search for super-narrow dibaryon resonances by the $p d \rightarrow p d X$ and $p d \rightarrow p p X$ reactions
A18(2003)2491

A18(2003)1145

A18(2003)2501

A18(2003)1499

A18(2003)1225

A18(2003)1215

A18(2003)1681

A18(2003)2509

A18(2003)2217

A18(2003)2311

A18(2003) 31

A18(2003) 143

A18(2003) 294

A18(2003)298

A18(2003) 327

A18(2003)440

A18(2003)402

A18(2003)1145

A18(2003)859

A18(2003)1485

A18(2003)1247

A18(2003)1247

A18(2003)151

A18(2003) 85

A18(2003)294

A18(2003) 102

A18(2003)139

A18(2003) 85

A18(2003) 147

A18(2003)362

A18(2003) 294

A18(2003)298

A18(2003) 313

A18(2003)327

A18(2003)410
Tamii, A., Uchigashima, N., Sakai, H., Hatano, M., Maeda, Y., Saito, T., Ishida, T., Kuboki, H., Hatanaka, K., Wakasa, T., Kamiya, J., Shimizu, Y., Kitamura, Y., Okamura, H., Suda, K., Yako, K., Sekiguchi, K. \& Satou, Y., Measurement of $p+d$ elastic scattering at $E_{P}=392 \mathrm{MeV}$

A18(2003)440

Tamura, H., Ajimura, S., Akikawa, H., Alburger, D.E., Banu, A., Chrien, R.E., Eugenio, P., Franklin, G.B., Franz, J., Gan, L., Hashimoto, O., Hayakawa, T., Hotchi, H., Imai, K., Kishimoto, T., Khaustov, P., May, M., Minami, S., Miura, Y., Miyoshi, T., Mizunuma, K., Nagae, T., Nakamura, S.N., Nakazawa, K., Okayasu, Y., Pile, P.H., Quinn, B.P., Rusek, A., Sasao, J., Sato, Y., Sawafta, R., Schmitt, H., Sutter, R., Takahashi, H., Tang, L., Tanida, K., Ukai, M., Yuan, L., Zhou, S.H., Zhu, L.H. \& Zhu, X.F., Gamma spectroscopy of $p$-shell hypernuclei and $\Lambda N$ spin-dependent interactions Report of BNL E930

Tanaka, K., see Kawamura

Tanaka, K., see Cleaver

Tang, L., see Tamura

Tang, L. (for Jefferson Lab E89009 Collab.), First experiment to produce $\Lambda$-hypernuclei using electron beam at Jlab

Tanida, K., see Tamura

Taniguchi, Y., see Watanabe

Tanimura, S., Quantization on a torus without position operators

Tarallo, M., see Morando

Teotonio-Sobrinho, P., see de Albuquerque

Ternovoi, V.Ya., see Fortov

Thomas, A.W., Leinweber, D.B., Young, R.D. \& Wright, S.V., QCD and hadron structure

Thomas, A.W., see Mineo

Thomas, A.W., Closing remarks

Thomas, A.W., see Detmold
A18(2003) 112

A18(2003) 85

A18(2003)799

A18(2003) 1743

A18(2003) 85

A18(2003) 85

A18(2003)182

A18(2003)2755

A18(2003)1841

A18(2003)2517

A18(2003) 1835

A18(2003)347

A18(2003) 384

A18(2003)456

A18(2003)2681 
Tian, Y., Zhu, C.-J. \& Song, X.-C., Topological charge of noncommutative ADHM instanton

Tohsaki, A., see Funaki

Toller, M., On the Lorentz transformations of momentum and energy

Tolstoy, V.N., see Borowiec

Toppan, F., see Carrion

Toppan, F., see Carrion

Tornow, W., Neidel, E.M., González Trotter, D.E., Howell, C.R., Crowell, A.S., Macri, R.A., Walter, R.L., Weisel, G.J., Esterline, J., Witała, H., Crowe III, B.J., Pedroni, R.S. \& Markoff, D.M., Electromagnetic effects and the long-standing three-nucleon analyzing power puzzle

Tornow, W., Czakon, N.G., Howell, C.R., Hutcheson, A., Kelley, J.H., Litvinenko, V.N., Mikhailov, S., Pinayev, I.V., Weisel, G.J. \& Witała, H., Analyzing power for the photodisintegration of the deuteron between $E_{\gamma}=2.4$ and 4.0 MeV

Torrielli, A., Unitarity of noncommutative field theories from string theory

Trivdei, G., A representation of symmetry generators for type IIB superstring on a plane wave in the U(4) formalism

Tsou, S.T. \& Palmer, J.S., Mass and mixing of fermions from a rotating mass matrix

Tsuruta, K., see Yagita

Tsuruta, K., see Ishida

Tsutsui, I., see Fülöp

Tuite, M. \& Sen, S., A string motivated approach to the relativisitc point particle

Turakulov, Z. \& Safonova, M., Motion of a vector particle in a curved spacetime.

I. Lagrangian approach

Turan, G., see Şirvanlı

Turan, G., see Eygi

Uccirati, S., Algebraic-numerical methods for evaluating multileg and multi-loop Feynman diagrams
A18(2003) 1691

A18(2003) 170

A18(2003)2019

A18(2003)1157

A18(2003)629

A18(2003) 787

A18(2003)258

A18(2003)282

A18(2003)2525

A18(2003)1317

A18(2003) 807

A18(2003) 322

A18(2003)436

A18(2003)2863

A18(2003)2483

A18(2003)579

A18(2003) 47

A18(2003)2735

A18(2003)2849
Uchai, W., see Yan

Uchigashima, N., see Saito

Uchigashima, N., see Maeda

Uchigashima, N., see Sekiguchi

Uchigashima, N., see Tamii

Uchigashima, N., see Tamii

Ueda, T., see Itonaga

Uesaka, T., see Saito

Uesaka, T., see Sekiguchi

Uesaka, T., see Tamii

Ukai, M., see Tamura

Uvarov, D.V., $N=2$ supersymmetric Yang-Mills theory and the superparticle: Twistor transform and $\kappa$-symmetry

van den Bossche, B., see Debergh van Neerven, W.L., see Ravindran Vaucher, A.R., see Desai

Vergara, J.D., see Romero

Vitale, P., see Lizzi

Volkov, G., see Anselmo

Voloshin, M.B., Remarks on the decays $\chi_{b J}^{\prime} \rightarrow \omega \Upsilon$

Voloshin, M.B., Variation of the relative yield of charged and neutral $B$ mesons across the $\Upsilon(4 S)$ resonance

Vuletić, V., see Chin

Vysotsky, M., $\mu_{v}$

Wakasa, T., see Maeda

Wakasa, T., see Shimizu

Wakasa, T., see Yagita

Wakasa, T., see Sekiguchi

Wakasa, T., see Tamii

Wakasa, T., see Tamii

Walter, R.L., see Tornow

Wang, B., Abdalla, E. \& Su, R.-K., Dynamics and holographic discreteness of tachyonic inflation

Wang, F., Ping, J.-L., Pang, H.-R. \& Goldman, T., Which constituent quark model is better?

Wang, J.S., see Zhang

Wang, K., see Zhang

Wang, M., see Zhang

Wang, W.Y., see Wu

Watabiki, Y., see Ookouchi

Watanabe, T., see Hayashi

Watanabe, T., Taniguchi, Y., Sawado, N. \& Oryu, S., Analysis of light nuclei with the AMD method

Weeks, J.R., Detecting topology in a nearly flat hyperbolic universe

Wei, Y.B., see Zhang
A18(2003) 370

A18(2003)294

A18(2003)298

A18(2003)327

A18(2003) 410

A18(2003)440

A18(2003)135

A18(2003)294

A18(2003)327

A18(2003)410

A18(2003) 85

A18(2003)1611

A18(2003) 1013

A18(2003)1721

A18(2003) 1355

A18(2003)1673

A18(2003)2381

A18(2003)699

A18(2003)1067

A18(2003)1783

A18(2003)398

A18(2003) 877

A18(2003)298

A18(2003) 313

A18(2003) 322

A18(2003)327

A18(2003)410

A18(2003)440

A18(2003)258

A18(2003)31

A18(2003)356

A18(2003)151

A18(2003)151

A18(2003)151

A18(2003)1303

A18(2003)1113

A18(2003)2785

A18(2003)182

A18(2003)2099

A18(2003)151 
Weisel, G.J., see Tornow

Weisel, G.J., see Tornow

Weller, H.R. \& Ahmed, M.W., The HI $\gamma$ S facility: A free-electron laser generated gamma-ray beam for research in nuclear physics

Williams, B.W. \& Lévai, G., An asymmetric "implicit" potential on the real line

Witała, H., see Tornow

Witała, H., see Tornow

Wright, S.V., see Thomas

Wu, K., see Zhou

Wu, Y.L., Yan, Y.A., Zhong, M., Zuo, Y.B. \& Wang, W.Y., HQEFT as a large component QCD and comments on the incompleteness of HQET

Xiao, G.Q., see Zhang

Xiao, Z.G., see Zhang

$\mathrm{Xu}$, H.S., see Zhang

Xue, S.-S., see Gurzadyan

Xue, S.-S., The dynamical Casimir effect and energetic sources for gamma ray bursts

Yagita, T., Sagara, K., Ishida, T., Minami, S., Tsuruta, K., Noro, T., Kamiya, J., Wakasa, T., Yoshida, H., Yoshimura, M., Hatanaka, K. \& Akiyoshi, H., Tensor anomaly in pd radiative capture

Yagita, T., see Ishida

Yako, K., see Saito

Yako, K., see Maeda

Yako, K., see Shimizu

Yako, K., see Sekiguchi

Yako, K., see Tamii

Yako, K., see Tamii

Yamada, T., see Hiyama

Yamamoto, Y., see Hiyama

Yamazaki, T., see Doté

Yan, M.-L., see Jiang

Yan, Y., Kobdaj, C., Uchai, W., Faessler, A., Gutsche, T. \& Zheng, Y.M., $e^{+} e^{-}$annihilation into $\bar{N} N$ pairs

Yan, Y.A., see Wu

Yang, K.-C., see Chua

Yang, S.N. \& Kamalov, S.S., Probing $\Delta$ structure with pion electromagnetic production

Yang, Z.-Y., see Chen

Yao, Q.-K., see Li
A18(2003) 258

A18(2003)282

A18(2003)1569

A18(2003)1901

A18(2003) 258

A18(2003) 282

A18(2003) 347

A18(2003)1911

A18(2003)1303

A18(2003) 151

A18(2003) 151

A18(2003) 151

A18(2003)561

A18(2003) 1325

A18(2003) 322

A18(2003)436

A18(2003)294

A18(2003) 298

A18(2003) 313

A18(2003) 327

A18(2003) 410

A18(2003)440

A18(2003)95

A18(2003) 95

A18(2003) 120

A18(2003)977

A18(2003) 370

A18(2003)1303

A18(2003)1763

A18(2003)248

A18(2003)2913

A18(2003) 2775
Yasuda, T., see Rodgers

Yazadjiev, S.S., Stiff perfect fluid singularity-free diagonal inhomogeneous cosmologies in scalar-tensor theories

Yazadjiev, S.S., A class of homogeneous scalar-tensor cosmologies with a radiation fluid

Yazadjiev, S.S. \& Rizov, V.A., Singularity free cosmological solutions of Einstein-Maxwell equations

Yazaki, K., see Mineo

Yi, L.-Z., see Jia

Yoshida, H., see Yagita

Yoshida, H.P., see Shimizu

Yoshimura, M., see Yagita

Yoshino, H., Harada, K., Matsuda, M. \& Nagata, J., Phase-shift analysis of $p-{ }^{3} \mathrm{He}$ scattering below $200 \mathrm{MeV}$

Yoshino, H., see Nagata

Young, R.D., see Thomas

$\mathrm{Yu}, \mathrm{H}$., see $\mathrm{Li}$

Yuan, L., see Tamura

Yuan, L.P., Chmielewski, K., Deltuva, A., Oelsner, M., Sauer, P.U. \& Adam, J., Jr., Two-body electro disintegration of the trinucleon bound state

Yue, C.X., Zong, H.J. \& Liu, L.J., Non-universal gauge bosons $Z^{\prime}$ and rare top decays

Zampini, A., see Lizzi

Zhan, W.L., see Zhang

Zhang, F.S. \& Lynden-Bell, R.M., Pure vibrational dephasing of triiodide in liquids and glasses

Zhang, H.Y., Shen, W.Q., Ma, Y.G., Cai, X.Z., Fang, D.Q., Zhong, C., Wei, Y.B., Chen, J.G., Zhou, X.F., Ma, G.L., Wang, K., Ren, Z.Z., Zhan, W.L., Guo,, Z.Y., Xiao, G.Q., Xu, H.S., Wang, J.S., Sun, Z.Y., Li, J.X., Wang, M., Chen, Z.Q., Xiao, Z.G., Li, W.F., Li, J.F., Hu, Z.G., Bai, J. \& Chen, L.X., Possible exotic structure in light proton-rich nuclei

Zhang, H.Y., see Ren

Zhang, P.-M., Duan, Y.-S. \& Cao, L.-M., The order parameter field for cosmic string, inflation and dark energy
A18(2003)2467

A18(2003)471

A18(2003)1967

A18(2003)2555

A18(2003)384

A18(2003) 1247

A18(2003) 322

A18(2003) 313

A18(2003)322

A18(2003) 444

A18(2003)448

A18(2003)347

A18(2003)2775

A18(2003) 85

A18(2003)244

A18(2003)2187

A18(2003)2381

A18(2003)151

A18(2003)406

A18(2003)151

A18(2003) 174

A18(2003)2587 
Zhang, X., see Piao

Zhang, Y. \& Su, R.-K., Stability of strangelets in the quark mass density- and temperaturedependent model

Zhang, Y.-Z., see Piao

Zhao, E., see Li

Zhao, E.-G., see Sun

Zheng, Y.M., see Yan

Zhong, C., see Zhang

Zhong, M., see Wu

\begin{tabular}{l|l} 
A18(2003)2109 & $\begin{array}{l}\text { Zhou, B., Guo, H.-Y., Pan, J. \& } \\
\text { Wu, K., The Euler-Lagrange } \\
\text { cohomology and general } \\
\text { volume-preserving systems }\end{array}$ \\
A18(2003)143 & $\begin{array}{l}\text { Zhou, S.H., see Tamura } \\
\text { Zhou, X.F., see Zhang }\end{array}$ \\
A18(2003)2109 & Zhu, C.-J., see Tian \\
A18(2003)2713 1485 & Zhu, L.H., see Tamura \\
A18(2003)370 & Zhu, X.F., see Tamura \\
A18(2003)151 & Zinovjev, G.M., see Molodtsov \\
A18(2003)1303 & Zong, H.J., see Yue \\
& Zuo, Y.B., see Wu
\end{tabular}

A18(2003)1911

A18(2003) 85

A18(2003)151

A18(2003)1691

A18(2003) 85

A18(2003) 85

A18(2003)817

A18(2003) 2187

A18(2003) 1303 